Monatsschr Kinderheilkd 2012 • 160:887-894 DOI 10.1007/s00112-012-2680-y

Online publiziert: 21. April 2012

(c) Springer-Verlag 2012

\section{Redaktion}

B. Koletzko, München

T. Lücke, Bochum

N. Wagner, Aachen

S. Wirth, Wuppertal

F. Zepp, Mainz

\author{
M. Boettcher ${ }^{1} \cdot$ S. Göttler ${ }^{1}$ L. Stoffel $\left.\right|^{2}$ K. Schwab ${ }^{2}$ St. Berger ${ }^{2} \cdot$ M. Mérat ${ }^{2}$ \\ ${ }^{1}$ Abteilung für Chirurgie, Universitätskinderkliniken, Inselspital Bern \\ ${ }^{2}$ Interdisziplinäres Schmerzfachteam, Universitätskinderkliniken, Inselspital Bern
}

\title{
Schmerzmanagement bei Kindern in der Schweiz
}

Morbidität, Mortalität, Angst und Anspannung von Patient, Angehörigen und Personal und letztlich die Behandlungskosten signifikant erhöht werden [25].

Aktuelle Studien belegten, dass stationär behandelte Kinder noch heute z. T. hohen Schmerzen ausgesetzt sind [14]. Tendenzielle Verbesserungen der Schmerztherapie in England, Italien und Österreich wurden durch nationale Umfragen festgestellt. Jedoch sind bestimmte Anteile der Schmerztherapie unterentwickelt, z. B. die prozedurale Schmerzbehandlung bei Neonaten [25].

In der Schweiz wurde die pädiatrische Schmerztherapie bisher nicht evaluiert. Ziel dieser Studie war es, den gegenwärtigen Umgang mit Schmerzen an Kinderkrankenhäusern in der Schweiz zu ermitteln. Weiterhin sollte das Vorhandensein von Leitlinien zur Erfassung und Therapie von Schmerzen überprüft werden.

\section{Methoden}

Die nationale Erhebung des Schmerzmanagements bei Kindern in der Schweiz wurde im Dezember 2009 durchgeführt. Der von den Autoren entwickelte Fragebogen wurde innerhalb des eigenen Klinikums getestet und anschließend an alle Krankenhäuser $(n=45)$ mit einer pädiatrischen Abteilung versandt. Kindernotfallstationen wurden nicht explizit angeschrieben. Der Fragebogen war semianonym und wurde mit frankiertem Rücksendeumschlag verschickt. Zur Verbesserung des Rücklaufs erhielten alle angeschriebenen Institutionen 3 Monate später ein Erinnerungsschreiben. Die Ergeb- nisse des eigenen Instituts wurden nicht berücksichtigt.

Der insgesamt 6 Seiten lange Fragebogen umfasst 42 Fragen, insbesondere Auswahlfragen und gelegentlich Freitext. Er gliedert sich in 4 Hauptthemen:

- demografische Daten,

- allgemeine Fragen,

- Schmerzerfassung und

- Schmerzmanagement.

Am Anfang des Fragebogens wird die Krankenhausstruktur inklusive Bettanzahl erfasst, wobei zwischen Universitätskrankenhäusern mit neonatologischer und intensivmedizinischer Abteilung, größeren kantonalen Kliniken und kleineren regionalen Häusern unterschieden wird.

Im zweiten Abschnitt zu den allgemeinen Fragen wird der Nutzen einer nationalen Plattform zum gemeinsamen Austausch über Schmerzen bei Kindern und die Häufigkeit von Weiterbildungen erfragt.

Die Fragen zum Themengebiet der Schmerzerfassung evaluieren die hierzu verwendeten Instrumente, wie die Anwendung von etablierten Skalen und Scores sowie das Vorhandensein von Leitlinien zur Interpretation der Resultate.

Im Abschnitt über das Schmerzmanagement werden beispielhaft typische $\mathrm{Si}$ tuationen der pädiatrischen Schmerztherapie bei Lumbal-, Knochenmarkpunktionen, Spickdrahtentfernungen und Versorgung von Riss-Quetsch-Wunden thematisiert, wie auch der Einsatz von $\mathrm{N}_{2} \mathrm{O}-\mathrm{O}_{2}$ und die patientenkontrollierte Analgesie (PCA). Die Morphiumapplikation wird ebenso erfasst wie auch die Kompetentes Schmerzmanagement bei Kindern können die Rekonvaleszenz verzögert und die 


\section{Originalien}

\begin{tabular}{|c|c|}
\hline \multirow[t]{2}{*}{ Demografische Fragen } & Krankenhauskategorie \\
\hline & Bettenzahl \\
\hline \multirow[t]{2}{*}{ Allgemeine Fragen } & Gemeinsame Schweizer Plattform \\
\hline & Interne Weiterbildungen zum Thema Schmerz \\
\hline \multirow[t]{2}{*}{ Schmerzerfassung } & Schmerzerfassungsinstrumente (Neonaten und Kinder) \\
\hline & Leitlinien zur systematischen Schmerzerfassung \\
\hline \multirow[t]{10}{*}{ Schmerzmanagement } & Einsatz von Lachgas-Sauerstoff-Gemisch \\
\hline & Schmerzbehandlung bei Lumbalpunktionen \\
\hline & Schmerzbehandlung bei Knochenmarkpunktionen \\
\hline & Schmerzbehandlung bei Spickdrahtentfernungen \\
\hline & Schmerzbehandlung bei Wundversorgungen \\
\hline & Schmerzbehandlung bei periphervenösen Zugängen \\
\hline & Anwendungsgebiete von PCA-Pumpen \\
\hline & Einsatz von Morphium \\
\hline & Kompetenzen von Pflegefachpersonen \\
\hline & Leitlinien in pädiatrischer Schmerztherapie \\
\hline \multirow[t]{2}{*}{ Postoperative Schmerztherapie } & Modalitäten \\
\hline & Applikation der Schmerzmedikamente \\
\hline \multirow{5}{*}{$\begin{array}{l}\text { Schmerzbehandlung } \\
\text { beim Frühgeborenen }\end{array}$} & Notfallmäßige endotracheale Intubationen \\
\hline & Elektive endotracheale Intubationen \\
\hline & Endotracheales Absaugen \\
\hline & Legen eines periphervenösen Zugangs \\
\hline & Kapillare Blutentnahmen \\
\hline \multirow{4}{*}{$\begin{array}{l}\text { Schmerzbehandlung } \\
\text { bei Kindern auf Intensivstationen }\end{array}$} & Endotracheales Absaugen \\
\hline & Legen eines zentralvenösen Zugangs \\
\hline & Schmerztherapie bei intubierten, beatmeten Kindern \\
\hline & Schmerztherapie bei operativen Eingriffen auf der Intensivstation \\
\hline
\end{tabular}

zen der Pflege bei der Schmerzmittelverabreichung. Des Weiteren wird das Management von postoperativen Schmerzen erfragt. Fragen bezüglich des Schmerzmanagements bei invasiven Prozeduren an Früh- und Neugeborenen auf Intensivstationen finden sich im Folgenden. Manipulationen bei Frühgeborenen wie elektive und Notfallintubationen, endotracheales Absaugen, Blutentnahmen und Kathetereinlagen werden ausführlich abgehandelt. Die weiteren Fragen befassen sich mit dem Umgang bei Schmerzen auf Intensivstationen in häufigen Situationen wie Intubation, Kathetereinlage und Beatmung. Nichtpharmakologische Methoden werden jeweils nicht detailliert abgefragt.

Die Themenüberschriften des Fragebogens sind in 1 Tab. 1 zusammengefasst.

Da die verschiedenen Facetten der pädiatrischen Schmerztherapie möglichst umfassend dargestellt werden sollten, wurden die Antworten deskriptiv aus-
Stationen setzen 48\% Tools ein. Am häufigsten kamen die visuelle Analogskala (VAS; 81\%), die Smiley-Skala (61\%), der Berner Schmerzscore für Neugeborene (BSN; 46\%) und die Gesichterskala nach Bieri (38\%) zum Einsatz. Die Verwendung der Instrumente war weitgehend von der Versorgungsebene unabhängig.

Zum Zeitpunkt der Befragung waren in $78 \%$ der Einrichtungen eine hausinterne oder extern publizierte Leitlinie (Universitätskliniken $100 \%$, restliche $69 \%$ ) zur Schmerzerfassung eingeführt.

\section{Schmerztherapie}

Die Mehrheit der befragten Institutionen (gesamt 78\%, Universitätskliniken $80 \%)$ behandelten Schmerzen aufgrund von hausinternen Leitlinien. In knapp 1/3 (30\%) der Einrichtungen war keine Leitlinie etabliert. Häufig (70\%) wurde im Falle von Schmerzen eine weitere Disziplin hinzugezogen, wobei überwiegend ein interdisziplinäres Schmerzteam (41\%) und die Anästhesie (52\%) konsultiert wurden.

Analgetika kamen immer bei prozeduralen Schmerzen zum Einsatz. Bei Lumbalpunktionen wurden v. a. lokale (74\%), topische Analgetika wie EMLA („eutectic mixture of local anesthetic", 96\%), Lachgasinhalation (83\%) und Sedativa (61\%) verwendet. Wundversorgungen erfolgten u. a. mit lokaler Analgesie (92\%), Sedativa wie Midazolam (46\%) oder $\mathrm{N}_{2} \mathrm{O}$ Inhalation (79\%). Bei schmerzhaften Eingriffen wie einer Knochenmarkpunktion und Metallentfernung erfolgte die Behandlung durch Lachgas (42\%), Midazolam (29\%) und nichtsteroidale Antiphlogistika (NSAID, 29\%). In allen Fällen wurde nicht nur sedierend, sondern auch analgetisch behandelt. Die Versorgungsebene hatte auf die Wahl der Schmerztherapie keinen wesentlichen Einfluss.

Bis auf 2 kleinere Kliniken verwendeten alle Einrichtungen patientenkontrollierte Analgesie (PCA). Sie wurde immer durch die Anästhesie und in einem Fall zusätzlich durch die onkologische Abteilung initiiert und betreut.

Pflegekontrollierte Analgesie wurde lediglich von einer Abteilung abgelehnt. Insgesamt befürworteten $57 \%$ die Verabreichung von Bedarfsmedikation nach ärztlicher Verordnung und 39\% nach Leit- 
linien. An Universitätskliniken sprachen sich 3/4 für eine pflegekontrollierte Behandlung nach Leitlinien aus.

Intravenöse Opioide wurden meist durch das ärztliche Personal verabreicht, bei 1/3 aller Kliniken auch durch das Pflegepersonal. 6 Einrichtungen - darunter eine Universitätsklinik - lehnten i.v. Opioidgaben durch das Pflegepersonal vollständig ab.

\section{Postoperative Schmerzen}

Sie wurden immer durch eine Form von Analgesie behandelt. Dabei kamen Nichtopioidanalgetika, wie Paracetamol, Ibuprofen, Diclofenac und Metamizol, oder Opioide, wie Morphium, Nalbuphin und Tramadol, nahezu gleich oft zur Anwendung. Insgesamt am häufigsten wurden Paracetamol (96\%), Morphium (81\%) und Diclofenac (70\%) verwendet. Die Applikation erfolgte überwiegend und gleich häufig per os, rektal oder i.v. Die parenterale, postinterventionelle Schmerztherapie war häufig (54\%) patientenkontrolliert. Es fand sich jeweils kein Unterschied zwischen den Versorgungsebenen.

\section{Neonatologie}

Bei invasiven Eingriffen wie Notfall- oder Elektivintubationen und endotrachealem Absaugen wurden auf den neonatologischen Stationen zu 88\% Analgetika oder Sedativa verabreicht. Bei Universitätskrankenhäusern lag die Rate mit 94\% marginal höher als bei den anderen Versorgungsebenen. Für elektive Intubationen kam immer eine Form der Schmerztherapie zur Anwendung. Im Notfall setzten $87 \%$ Analgesie bei Intubationen ein. Bei den invasiven Interventionen wie Intubationen oder endotrachealem Absaugen wurden häufig Opioide (72\%) verwendet. 14\% der Befragten waren der Meinung, dass Morphin bei Neugeborenen keine Verwendung finden soll, und 30\% befürworteten einen zurückhaltenden Umgang des Medikaments bei Kindern.

Bei kleineren Eingriffen wie Venenpunktionen oder kapillaren Blutentnahmen wurden zu 92\% Analgesie und Sedierung verwendet. Im Einsatz waren häufig topische Analgetika (31\%) oder Zuckerlösungen (74\%). Unter den Zuckerlösungen

Monatsschr Kinderheilkd 2012 • 160:887-894 DOI 10.1007/s00112-012-2680-y

(c) Springer-Verlag 2012

\section{Boettcher · S. Göttler · L. Stoffel · K. Schwab · St. Berger · M. Mérat Schmerzmanagement bei Kindern in der Schweiz}

\section{Zusammenfassung}

Hintergrund. Schmerzen bei stationär aufgenommenen Kindern werden häufig unzureichend behandelt. Bisher gab es keine Informationen zum Schmerzmanagement von Kinderkrankenhäusern in der Schweiz. Ziel der vorliegenden Studie war, den aktuellen Stand der Schmerzerfassung, -interpretation und -behandlung zu bestimmen.

Studiendesign. Ein Fragebogen wurde an alle pädiatrischen Krankenhäuser in der Schweiz gesendet.

Ergebnisse. Insgesamt antworteten 27 von 45 Einheiten (Antwortrate: 60\%). Die meisten Abteilungen verwenden Schmerzerfassungstools (96\%) und führten diesbezügliche Leitlinien ein (78\%). Die Behandlung von Schmerzen erfolgt ebenfalls meist nach hausinterner Leitlinie (78\%). Prozedurale und postoperative Schmerzen werden stets (100\%) analgetisch behandelt. Bei Frühgeborenen und
Kindern auf Intensivpflegestationen werden bei invasiven Eingriffen häufig Analgetika (>87\%) verwendet. Auf Intensivstationen liegen in 44\% diesbezügliche Leitlinien vor. Resümee. Der Nutzen eines effektiven Schmerzmanagements bei Kindern ist eindeutig belegt. Viele Ansätze zur Verbesserung werden in der Schweiz gut umgesetzt. Vor allem im internationalen Vergleich verbesserte sich das Schmerzmanagement. Es gibt aber noch Optimierungsmöglichkeiten. Beispielsweise besitzen weniger als die Hälfte aller schweizerischen Intensivstationen eine Leitlinie für die Behandlung von Schmerzen bei invasiven Eingriffen.

Schlüsselwörter

Pädiatrie · Schmerzen .

Schmerzmanagement · Leitlinien · Schweiz

\section{Pain management for children in Switzerland}

\section{Abstract}

Objective. Pain in hospitalized children is often undertreated. The aim of the study was to assess the current pain management for children in Switzerland.

Study design. A postal questionnaire was sent to all pediatric units in Switzerland.

Result. A total of 27 of 45 units replied $(60 \%$ response). Most units used tools for pain assessment (96\%) and had a guideline for pain management (78\%). Procedural and postoperative pain are always $(100 \%)$ treated. Premature infants and children in intensive care units often $(>87 \%)$ receive analgesics during invasive procedures. However, only $44 \%$ of intensive care units have a guideline for this. Conclusion. Benefits of an effective pain management in children are well established. Many measures have been applied in Switzerland. However, there is still room for improvement, e.g., less than half of all intensive care units rely on guidelines for analgesics during invasive procedures.

\section{Keywords}

Pediatrics · Pain · Pain management . Guidelines · Switzerland lag 30\%ige Glukoselösung mit 35\% vor 20\%iger Glukose-(23\%), 20\%iger Saccharose- (21\%) und 63\%iger Zuckerlösung (3\%). Die einzelnen Abteilungen wiesen eindeutige Präferenzen auf, auf welche die Versorgungsebene keinen Einfluss hatte.

Auf den neonatologischen Stationen arbeiteten durchschnittlich $71 \%$ der befragten Institutionen mit einer Leitlinie. Universitäre Häuser verwendeten Leitlinien etwas häufiger (77\%) als kommunale und regionale Kliniken.

\section{Pädiatrische Intensivstation}

Auf intensivmedizinischen Stationen wurde Analgesie bei der Anlage eines zentralen Venenkatheters (ZVK) und bei endotrachealem Absaugen in $87 \%$ der Kliniken eingesetzt, wobei überwiegend Opioide (75\%) mit eindeutiger Präferenz für Morphium, vereinzelt in Kombination mit Midazolam, verwendet wurden. Zuckerlösungen fanden bei Kindern auf Intensivstationen bei Anlage eines ZVK oder beim Absaugen keine Verwendung. Schmerzmedikation wurde bei maschinell 
Hier steht eine Anzeige.

Springer 
Hier steht eine Anzeige.

第 Springer 
beatmeten Patienten in 75\% der Einrichtungen kontinuierlich, in $17 \%$ intermittierend und in $8 \%$ bei Bedarf gegeben. Universitätskliniken verabreichten die Analgesie stets kontinuierlich. Bei Verbrennungspatienten erfolgte die Schmerzapplikation $\mathrm{zu} 61 \%$ kontinuierlich, zu $8 \%$ intermittierend und $\mathrm{zu} 31 \%$ bei Bedarf.

Eine Leitlinie zur Scherzbehandlung hatten insgesamt $44 \%$ der Intensivstationen eingeführt. An Universitätskliniken waren es mit $43 \%$ etwas weniger.

\section{Weiterbildung}

Fortbildungen fanden bei $38 \%$ der Einrichtungen mehrmals pro Woche, bei $31 \%$ einmal wöchentlich, bei $26 \%$ selten und bei einer Institution überhaupt nicht statt. Auf universitärer Ebene waren deutlich mehr Lehrveranstaltungen zu verzeichnen. Alle Universitätskliniken führten entweder mehrmals oder einmal pro Woche Schmerzfortbildungen durch.

\section{Diskussion}

Ein Kind kann während eines Klinikaufenthaltes insbesondere auf akutmedizinischen, neonatologischen und intensivmedizinischen Stationen mehrmals täglich Schmerzen ausgesetzt sein [14]. Die Komplexität der Schmerzwahrnehmung bei Kindern ist bekannt und eine der größten Herausforderungen für die behandelnden Fachpersonen [25]. Wegen der später möglichen Auswirkungen eines unzureichenden Schmerzmanagements gibt es zahlreiche Bemühungen, die Qualität der Versorgung zu verbessern.

\section{Schmerzerfassung}

Eine der wichtigsten Maßnahmen ist die standardisierte, routinemäßige Schmerzerfassung. Die Beurteilung des Schweregrads von Schmerzen bei Kindern stellt eine anspruchsvolle Aufgabe dar. Kleinkinder, insbesondere Neonaten und entwicklungsverzögerte Kinder, äußern ihre Beschwerden selten verbal und ihre Reaktionen auf Schmerzen sind für den Betrachter nicht immer nachvollziehbar [9, 10]. Schmerzen werden daher bevorzugt mit selbsteinschätzenden Tools objektiviert. Fremdeinschätzungen durch Ver- haltensbeobachtung werden komplementär oder bei Neonaten und Kindern unter 7 Jahren angewendet [18, 23]. Hierbei besteht die Gefahr, dass die subjektive Wahrnehmung und Erfahrung des Betrachters unbewusst in die Beurteilung mit einfließen. Deshalb wird versucht, allgemeingültige und international vergleichbare Schmerzerfassungsinstrumente zu entwickeln. Bis heute wurden allein für Kleinkinder über 30 verschiedene Instrumente validiert, doch bislang gibt es keinen Goldstandard [7].

In der vorliegenden Studie gaben fast alle Einheiten an, mindestens ein Schmerzerfassungstool regelmäßig zu verwenden. In älteren Studien über die Schmerzerfassung, überwiegend bei Neonaten, wie in einer australischen Umfrage aus dem Jahr 2006, verwendeten nur $6 \%$ regelmäßig Schmerzerfassungstools [11]. 2 Jahre später zeigte eine groß angelegte Umfrage aus England, nach Einschätzung der Autoren, geringfügig bessere Ergebnisse [14]. Eine kanadische Untersuchung, aus dem gleichem Jahr, kam immerhin auf 27\% [23]. Die Ergebnisse unserer aktuellen Studie sind erstaunlich gut. Vermutlich spiegeln sie die strukturellen Unterschiede der medizinischen Versorgung wider. Möglicherweise wirkten sich auch die schlechten Werte der Vergangenheit und die damit verbundene Aufmerksamkeit für die Schmerzerfassung positiv auf die heutige Datenlage aus.

\section{Peri- und postinterventionelle Schmerztherapie}

In den letzten Jahren nahm die Anzahl diagnostischer und therapeutischer Eingriffe bei Kindern stetig zu. Entsprechend bedeutend ist die adäquate, möglichst multimodale Behandlung von prozeduralen und postoperativen Schmerzen. Je nach Art der Intervention und dem Schmerzempfinden des Kindes kann optimales Schmerzmanagement durch Sedierung und Anästhesie oder durch verhaltenspsychologische Maßnahmen mit weniger oder beinahe ohne pharmakologische Unterstützung erzielt werden [5].

Für die meisten Prozeduren wird lokale Analgesie allein oder in Kombination mit systemischer Analgesie eingesetzt [24]. Bei invasiveren Eingriffen, wie bei einer Lumbalpunktion, können Sedativa die periprozedurale Anspannung reduzieren und die Kooperation bei den folgenden Eingriffen wesentlich verbessern [1]. Bei interventionellen Schmerzen werden, anders als in der vorliegenden Studie, überwiegend Opioide und nichtsteroidale Antiphlogistika eingesetzt [15], während Kliniken in der Schweiz häufiger auf Lachgasinhalationen zurückgreifen. Die analgosedierende Wirkung des $\mathrm{N}_{2} \mathrm{O}$ ist gut steuerbar und kann bei Kindern aller Altersstufen sicher eingesetzt werden [26]. Diese Vorliebe für Lachsgasinhalationen könnte mit der Ausbildung des Pflegepersonals und Ressourcen in der Schweiz zusammenhängen.

Insgesamt wurden die allgemeinen Empfehlungen, wie unsere Studie zeigt, in Schweizer Kinderkliniken relativ gut umgesetzt. Das Verständnis für eine analgetische Behandlung bei prozeduralen und postoperativen Schmerzen scheint gut.

\section{Neonatologie und pädiatrische Intensivstationen}

Frühgeborene erleiden häufig auf neonatologischen oder intensivmedizinischen Abteilungen Schmerzen. Obwohl es heute keine mehr Zweifel gibt, dass auch Extremfrühgeborene Schmerzen empfinden, scheinen Neonaten weiterhin unterversorgt zu sein [8]. Bei endotrachealen Intubationen wurde in England und Irland (6\%), in Kanada (14\%) und in Frankreich (38\%) Analgesie verwendet $[6,8,11]$. Im deutschsprachigen Raum wurden 2007 bei $40 \%$ der Notfallintubationen und bei 83\% der elektiven Intubationen Schmerzmittel verabreicht [1]. 2008 setzten in England bereits 70\% Analgesie ein [14]. In unserer aktuellen Studie erhöhte sich die Rate auf $87 \%$ bei Notfall- und auf $100 \%$ bei elektiven Intubationen. Entgegen früherer Studien zeigen unsere Daten, dass Frühgeborene bei schmerzhaften Eingriffen, wie endotrachealem Absaugen, mehr Analgesie erhalten als Patienten auf pädiatrischen Intensivstationen. Dies könnte Folge des zunehmenden Wissens um die Schmerzempfindlichkeit von Neonaten und der damit einhergehenden Sensibilisierung sein. Diese Entwicklung setzte sich auch bei den häufig durchgeführten, kleineren, aber schmerzhaften Eingriffen, 
wie Blutabnahmen, fort. Vor einigen Jahren verwendeten hierbei in England lediglich 5\% und in Australien nur 15\% Analgesie [12, 17]. 2008 kamen englische Kinderkrankenhäuser bereits auf 35\% [14]. Gemäß unseren Daten finden diese Maßnahmen in der Schweiz bereits in $92 \%$ regelmäßig unter Analgesie statt.

Opioide bieten bei prozeduralen Schmerzen auf neonatologischen Stationen exzellente analgetische Wirkung und eine große therapeutische Breite. In der Vergangenheit verhinderte v. a. die Angst vor respiratorischer Depression und möglicher Abhängigkeit ihren breitflächigen Einsatz. Jedoch wird die Überlebensrate von Neonaten durch ihren Einsatz, anders als früher angenommen, nicht negativ beeinflusst [26]. In unserer Studie wurden sie mit $72 \%$ ähnlich häufig eingesetzt wie in vorhergehenden Studien [1]. Auch die Verwendung von Lokalanästhetika ist sicher und effektiv [12]. Dennoch kamen sie laut älteren Studien nur selten (12\%) zum Einsatz [14]. In unserer Umfrage verwendeten 37\% topische Analgesie.

Zuckerlösungen als Analgosedierung bei Neonaten sind nicht unumstritten. Sie wurden überwiegend als effektiv, günstig und sicher bewertet [19]. Allerdings konnte in experimentellen Untersuchungen wie in einer randomisierten, placebokontrollierten Studie von 2010 keine Veränderungen der neonatalen Gehirnaktivität oder spinalen Nozizeption festgestellt werden [16]. Sie wurden in der Schweiz mit $72 \%$ mehr als doppelt so häufig wie im Jahr 2008 in England eingesetzt [14].

\section{Leitlinien und Fortbildung}

Die Einführung von Leitlinien und regelmäßigen Fortbildungen, insbesondere auf intensivmedizinischen Abteilungen mit großen personellen Fluktuationen, tragen entscheidend dazu bei, die Erkenntnisse aus der Forschung in die Praxis umzusetzen $[4,14]$. Dennoch sind nur auf $78 \%$ aller befragten Einheiten Leitlinien zur Erfassung und Behandlung von Schmerzen eingeführt. Auf neonatologischen Stationen hatten $71 \%$ eine Leitlinie implementiert. In vorhergehenden Erhebungen, wie 2004 in Australien (15\%), 2006 in Frankreich (65\%), 2007 im deutschsprachigen Raum (44\%) und 2008 in Großbritan- nien (35\%), war dies jedoch noch seltener der Fall $[8,13,14]$. Erstaunlich gering war die Rate an Leitlinien auf Intensivstationen. Lediglich $44 \%$ aller Einheiten in der Schweiz hatten eine Leitlinie zur Behandlung von Schmerzen eingeführt.

$\mathrm{Zu}$ beachteten ist, dass sich die Qualität der Schmerzbehandlung nicht allein durch die Einführung von Leitlinien und durch deren Kenntnis verbessert [22]. Weitere Schritte wie regelmäßige Fortbildungen sind notwendig, um die Behandlung nachhaltig zu verbessern [13]. In der Schweiz wird dies bereits sehr gut gehandhabt. Gemäß unserer Umfrage fand Schmerzedukation in 69\% der Kinderkliniken mindestens einmal wöchentlich statt.

\section{Pflegekontrollierte Analgesie}

Ein weiterer Ansatzpunkt für Verbesserungen sind Techniken wie die PCA, die v. a. bei Kindern älter als 5 Jahre etabliert sind [5]. Die Ausweitung dieses Verfahrens auf jüngere Patienten und Kinder, die physisch oder kognitiv beeinträchtigt sind, ist durch entwicklungspsychologische und körperliche Limitierungen nur begrenzt möglich. In der Vergangenheit wurde in diesen Fällen vorwiegend auf kontinuierliche oder intermittierende Medikation zurückgegriffen, die Schmerzspitzen schlecht abfangen oder zu latenter Unterversorgung führen [22]. In den letzten Jahren wurde das Behandlungskonzept adaptiert und eine kontinuierliche Schmerzmittelgabe mit pflege- oder elternkontrollierten Boli („nurse-controlled analgesia“, NCA) bei Schmerzspitzen eingeführt [13].

Die Weiterführung dieses Ansatzes ist eine pflegekontrollierte und -indizierte Schmerzmittelabgabe in definierten Situationen. Insbesondere bei standardisierten Eingriffen könnte eine speziell ausgebildete Pflegekraft die Schmerzbehandlung entsprechend einer Leitlinie initiieren bzw. anpassen. Die Befürchtung, dass durch frühzeitige, arztunabhängige Schmerzmittelgabe die Diagnostik leiden könnte, bestätigte sich nicht [7]. Dennoch sprechen sich nur $39 \%$ der pädiatrischen Abteilungen für eine leitliniegesteuerte, pflegekontrollierte Analgesie aus. Vermutlich ist dies v. a. eine Frage der Verantwortlichkeit und Kompe- tenz des Pflegepersonals, die es einheitlich zu klären gilt.

Bias

Bei einer Rücklaufquote von $60 \%$ gelten übliche Limitierungen. Trotz des guten Ergebnisses für eine Briefumfrage können gewisse Verzerrungen nicht ausgeschlossen werden. Erschwerend kommt hinzu, dass nicht alle Versorgungseinheiten gleichmäßig oft antworteten. Die Besprechungspunkte des Fragebogens basieren auf einzelnen Themen und haben nicht den Anspruch, die Fragen zum Gebiet des pädiatrischen Schmerzkonzepts vollständig abzudecken. Einen entscheidenden Einfluss könnte auch die Beantwortung des Fragebogens durch eine einzelne Person pro Einheit gehabt haben.

\section{Fazit für die Praxis}

Der Nutzen eines effektiven Schmerzmanagements bei Kindern ist eindeutig belegt. Der Umgang mit pädiatrischen Schmerzen ist in der Schweiz, v. a. im internationalen Vergleich, relativ gut. Schmerzen bei Kindern werden meistens wahrgenommen und häufig behandelt. Viele Kinderkliniken in der Schweiz verfügen über Leitlinien zur Schmerzerfassung und -behandlung. Jedoch besitzen weniger als die Hälfte aller schweizerischen Intensivstationen eine Leitlinie für die Behandlung von Schmerzen bei invasiven Eingriffen.

Weitere Verbesserungen sind notwendig und können insbesondere im Bereich der Schmerzerfassung erzielt werden. Um das relativ hohe Niveau weiter zu verbessern, ist wichtig von einem "view from the health care practitioner" zu einem "view from child's experience" zu kommen. Unserer Ansicht nach würde eine nationale Leitlinie zur Erfassung und Behandlung von Schmerzen bei Kindern den Austausch und die Diskussion bedeutend erleichtern und sollte in naher Zukunft diskutiert werden. Ideal wäre eine einheitliche, aber altersentsprechende Interpretation und Erfassung von Schmerzen, auf die eine standardisierte und automatisierte Therapie folgt. 
